\title{
Who Uses Osteopathic Manipulative Treatment? A Prospective, Observational Study Conducted by DO-Touch.NET
}

Jane C. Johnson, MA; Brian F. Degenhardt, DO

From the A.T. Still Research Institute (Ms Johnson and Dr Degenhardt) and the Department of Osteopathic Manipulative Medicine at the A.T. Still University Kirksville College of Osteopathic Medicine (Dr Degenhardt) in Missouri.

Financial Disclosures: None reported.

Support: The current study was funded by a grant from the American Osteopathic Association (grant No. 11-04-

634).

Address correspondence to

Jane C. Johnson, MA, A.T. Still Research Institute, A.T. Still University, $800 \mathrm{~W}$ Jefferson St, Kirksville, MO, 63501-1443.

Email: jjohnson@atsu.edu

Submitted

November 30, 2018; revision received January 28, 2019; accepted February 15, 2019.
Context: Information about the characteristics of patients who use osteopathic manipulative treatment (OMT) is limited.

Objective: To determine the scope of conditions being managed with OMT and describe the characteristics of patients who receive OMT.

Methods: Researchers conducted a longitudinal, observational study on the use and effectiveness of OMT at 17 clinics where clinicians (ie, osteopathic and allopathic physicians and Canadian-trained osteopaths) provided OMT. Adult patients receiving OMT completed questionnaires immediately before, immediately after, and daily for 7 days after treatment. Data collected from patients included demographic information, chief complaint(s) and their severity, and health-related quality of life. Physical examination findings, treatment, and medical diagnosis documentation were extracted from medical records. Census data were used to assess whether patients were representative of the population of the county where the clinic was located.

Results: Data were collected from 927 patients at 1924 office visits. A majority of patients were women (690 [75\%]), white (854 [96\%]), and not Hispanic or Latinx (707 [95\%]). The mean (SD) age was 51.9 (15.9) years. When compared with census data, the sample had higher percentages of women, people aged 65 years and older, people who identified as white, people who were high school and college graduates, and people with higher household incomes than that of the county population. The most common chief complaints from patients were pain or discomfort in the lower back (311 [34\%]) and neck (277 [30\%]), which corresponded with the most common medical diagnoses. Patients reported that OMT, surgery, and medications were the most helpful treatments they had used previously for their chief complaint(s). Before receiving OMT, patients' health-related quality of life was significantly worse $(P \leq .05)$ than that of the general US population.

Conclusions: Adult patients receiving OMT are being treated primarily for musculoskeletal pain conditions, are not representative of the population of the county where the clinic was located, and have worse health-related quality of life than that of the general population. Information about the characteristics of patients who use OMT is important for defining osteopathic distinctiveness and identifying potential areas for increasing the use of OMT. (ClinicalTrials.gov number NCT02395965)

J Am Osteopath Assoc. 2019;119(12):802-812

doi:10.7556/jaoa.2019.133

Keywords: chronic pain, low back pain, OMT, osteopathic manipulative treatment 
$\mathrm{P}$ revious studies describing the characteristics of patients receiving osteopathic manipulative treatment (OMT) combined this form of manual therapy with other manual therapy modalities (eg, chiropractic, massage therapy), ${ }^{1-3}$ with other practitioner-based therapies (eg, acupuncture, naturopathy), ${ }^{4,5}$ or with all complementary and alternative medicine (CAM) approaches as defined by the National Institutes of Health-National Center for Complementary and Integrative Health. ${ }^{6,7}$ Osteopathic and chiropractic manipulation are the fourth most commonly used CAM approaches in the US and the most commonly used to treat illness. ${ }^{1,7}$ A US survey ${ }^{8}$ estimated $22.0 \%$ of adults have seen an osteopathic physician for health care needs, but not necessarily for OMT. As such, the problem with combining disparate modalities into a single heterogeneous group is that unique characteristics of patients who receive any single modality like OMT could be obscured.

DO-Touch.NET, a practice-based research network, was formed to evaluate and advance the practice of osteopathic manipulative medicine. Building on a preliminary study, ${ }^{9}$ the current longitudinal, prospective, observational study was designed to examine the use and effectiveness of OMT in real-world clinical settings. Our objectives were to determine the scope of conditions currently being treated with OMT, describe the characteristics of patients who receive OMT, compare the characteristics of these patients with the population characteristics of the county where they received treatment, determine which treatments and activities patients previously found most and least helpful for their chief complaint(s), and compare the quality of life of patients who received OMT with that of the general US population.

\section{Methods}

Researchers conducted a longitudinal, prospective, observational study on the use and effectiveness of OMT at clinics where clinicians (osteopathic and allopathic physicians and a Canadian-trained osteopath) provided
OMT (ClinicalTrials.gov number NCT02395965). Patients aged 18 years and older scheduled to receive OMT were recruited to participate when they arrived for their appointment. Exclusion criteria included patients who had difficulties communicating in English, dementia, or psychological conditions that could affect their ability to provide accurate information, or were research team members or employees of participating clinics. Local institutional review boards (A.T. Still University-Kirksville, Capital Region Medical Center, Midwestern University-Downers Grove, Midwestern University-Glendale) approved the study. Each patient signed an approved informed consent form before participation.

Patients completed a series of questionnaires, which included questions pilot tested during a preliminary study ${ }^{9}$ that were administered in electronic or paper format depending on patient preference. Patients completed questionnaires electronically directly in the database, which was managed using 2 different data collection tools: Assessment Center during 2011-2012 and REDCap ${ }^{10}$ hosted at A.T. Still University during 2013-2014. Patients mailed completed paper questionnaires to the DO-Touch.NET coordinating center using provided postage-paid envelopes, and questionnaire responses were entered into the database twice to ensure data quality. Data from patients were collected immediately before seeing their clinician, immediately after OMT, and daily for 7 days after OMT (Figure 1) Documentation of the somatic dysfunction diagnosed, OMT techniques used, medical diagnoses, and treatment plan and home instructions was extracted from medical records.

Average and worst severity of the chief complaint(s) were evaluated using an 11-point numerical rating scale $(0=$ no problem to $10=$ worst imaginable). Assessment of the severity of the chief complaint(s) included the frequency and duration of symptoms. Health-related quality of life was assessed using customized National Institutes of Health Patient-Reported Outcomes Measurement System (PROMIS) fatigue, pain interference, physical functioning, and sleep disturbance short forms 


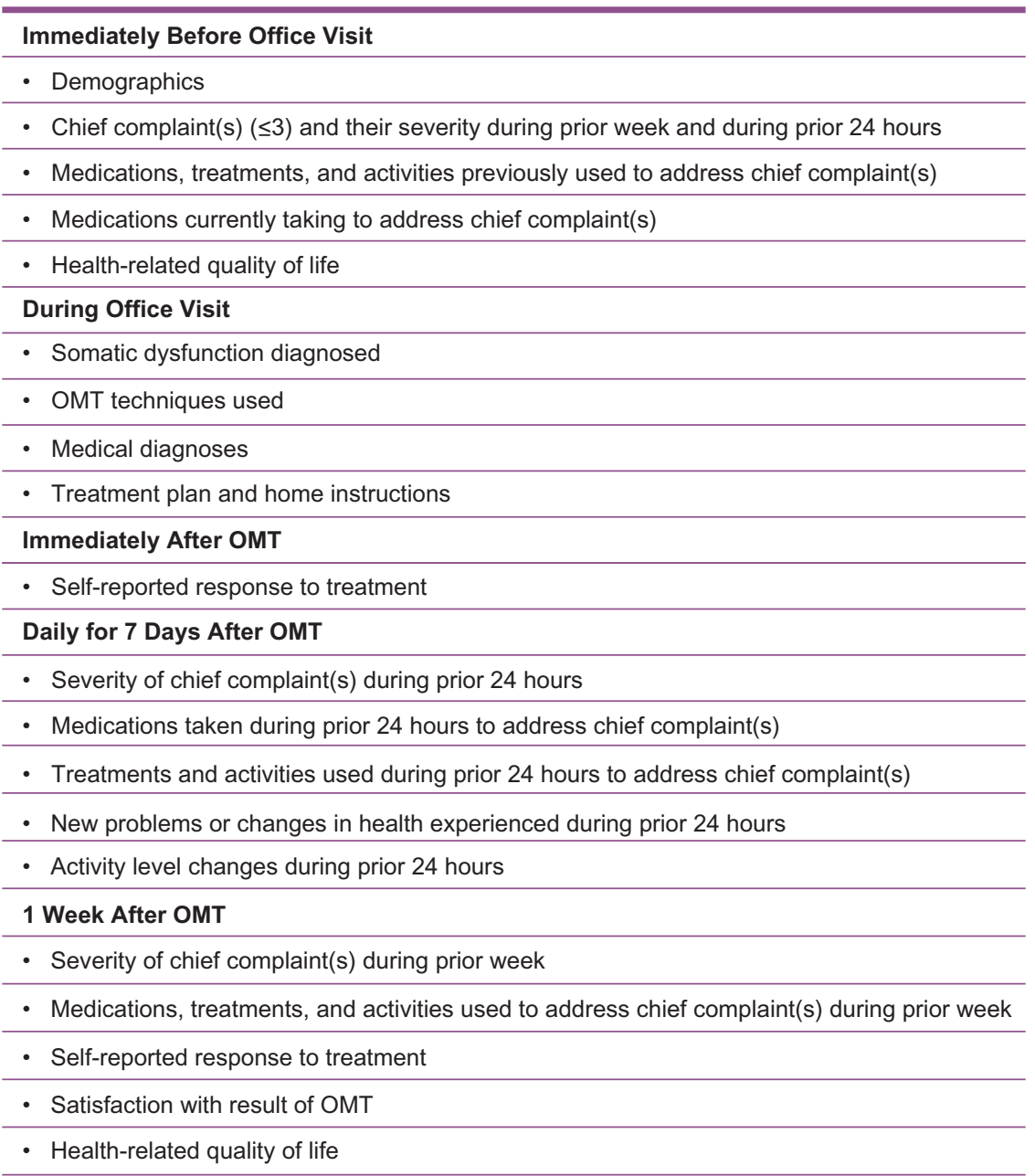

Figure 1.

Data collection timeline for the current longitudinal, prospective, observational study on the use and effectiveness of osteopathic manipulative treatment (OMT).

(http://www.healthmeasures.net/explore-measurementsystems/promis). The HealthMeasures Scoring Service (https://www.assessmentcenter.net/ac_scoringservice) was used to convert the raw scores from the PROMIS scales into $t$ scores. Conversion to $t$ scores facilitated comparison of patients' quality of life with the general US population, which has a mean (SD) $t$ score of 50 (10). ${ }^{11} t$ scores higher than 50 indicate worse fatigue, pain interference, and sleep disturbance, and $t$ scores lower than 50 indicate worse physical functioning than that of the general population.
Self-reported responses to treatment assessed how patients felt immediately after the clinician had left the treatment room and 1 week after OMT compared with before their OMT session by using a 5-point ordinal scale (much better, better, about the same, worse, much worse). When patients indicated that they felt better or worse than before OMT, they were asked to describe how their condition had changed. Satisfaction with the result of OMT was assessed using a 6-point ordinal scale (completely satisfied, very satisfied, somewhat satisfied, 
somewhat dissatisfied, very dissatisfied, completely dissatisfied).

Groupings of chief complaints and medical diagnoses were defined by consensus of 2 research team members (J.C.J. and B.F.D.). Demographic data, chief complaints, medical diagnoses, and previous treatments and activities used to address chief complaints were summarized using the frequency and percentage of patients who responded to the specific item, the median, and the mean (SD). Census data on age, gender, race, ethnicity, education, disability, and household income were used to assess whether patients were representative of the population of the county where the clinic was located. ${ }^{12}$ For each clinic with more than 10 patients, the percentage of patients from that clinic in the demographic categories of the census data and the median annual household income were calculated.

Survey sampling methods with sampling weights based on number of patients from the clinic were used to calculate a $95 \%$ CI for the mean difference between the sample statistics for each clinic and population statistics for the corresponding county. Confidence intervals that did not include a difference of 0 were considered to indicate a significant difference between the sample demographic composition and the population of the county where the clinic was located. General linear mixed models were fit to the PROMIS $t$ scores, allowing for the correlation of scores between office visits for each patient, to estimate the mean and 95\% CI for health-related quality of life. Since the mean $t$ score for the general US population was 50 , CIs for the mean $t$ score that did not include a $t$ score of 50 were considered to indicate a significant difference in health-related quality of life of the patients from that of the general US population.

\section{Results}

Forty-one DO-Touch.NET clinicians (39 osteopathic physicians, 1 allopathic physician, and 1 Canadiantrained osteopath; 17 specialized in osteopathic manipulative medicine, 12 were primary care providers, and 12 were board certified in both) from 17 clinics provided patients for the study. Data were collected from 927 patients at 1924 office visits. A total of 644 patients $(70 \%)$ contributed data from 1 office visit, 132 (14\%) from 2 office visits, 49 (5\%) from 3 office visits, 34 (4\%) from 4 office visits, and 68 (7\%) from 5 or more office visits. The number of patients per clinician ranged from 1 to 114 (median, 40). The mean (SD) age was 51.9 (15.9) years (range, 18-98 years) (Table). Most patients were women (690 [75\%]), white (854 [96\%]), and not Hispanic or Latinx (707 [95\%]). Six hundred eighty-eight patients $(85 \%)$ had some posthigh school education, 474 (58\%) were employed full time or part time, and the median annual household income was between $\$ 50,000$ and $\$ 74,999$.

Fifteen clinics $(88 \%)$ had more than 10 patients and were included in the county comparison analysis. Based on the $95 \% \mathrm{CI}$, the sample of patients who participated in the study had a significantly higher percentage of women (mean difference, 22\%; 95\% CI, $11 \%-33 \%$ ) and people aged 65 years and older (mean difference, $13 \%$; $95 \% \mathrm{CI}, 1 \%-25 \%$ ) than that of the county population. The sample had a significantly higher percentage of patients who identified as white (mean difference, 12\%; 95\% CI, 5\%-19\%) and a lower percentage of people who identified as black or African American (mean difference, $-9 \%$; 95\% CI, $-15 \%$ to $-4 \%$ ), Asian (mean difference, $-3 \% ; 95 \%$ CI, $-7 \%$ to $-0 \%$ ), Native Hawaiian or Pacific Islander (mean difference, $-0.1 \% ; 95 \% \mathrm{CI},-0.3 \%$ to $-0.0 \%$ ), and Hispanic or Latinx (mean difference, $-9 \%$; 95\% CI, $-15 \%$ to $-4 \%$ ) than that of the county population. No significant difference was found between the sample and county population in the percentage of people who identified as American Indian or Alaska Native (mean difference, $-0.2 \%$; $95 \% \mathrm{CI},-1.2 \%$ to $0.8 \%$ ) or as a member of more than 1 race (mean difference, $-0.2 \%$; $95 \% \mathrm{CI},-3.1 \%$ to $2.7 \%$ ). The percentage of high school graduates (mean difference, $9 \%$; 95\% CI, $5 \%-13 \%$ ) and college graduates (mean difference, $25 \%$; $95 \% \mathrm{CI}, 12 \%-37 \%$ ) in the sample was significantly higher than that of the county population. No 
Table.

Demographic Characteristics of Adult Patients Receiving Osteopathic Manipulative Treatment $(\mathbf{N}=927)^{a}$

\begin{tabular}{lc}
\hline Demographic Characteristic & \\
\hline Age, y, Mean (SD) & $51.9(15.9)$ \\
\hline Gender & $690(75)$ \\
\hline Female & $225(25)$ \\
\hline Male & 12 \\
\hline Declined to respond & $854(96)$ \\
\hline Race & $9(1)$ \\
\hline White & $6(1)$ \\
\hline American Indian/Alaska Native & $4(<1)$ \\
\hline Asian & $1(<1)$ \\
\hline Black/African American & $9(1)$ \\
\hline Native Hawaiian/Pacific Islander & $6(1)$ \\
\hline More than 1 race & 38 \\
\hline Other &
\end{tabular}

\section{Ethnicity}

\begin{tabular}{|c|c|}
\hline Hispanic or Latinx & $35(5)$ \\
\hline Not Hispanic or Latinx & $707(95)$ \\
\hline Declined to respond & 185 \\
\hline \multicolumn{2}{|l|}{ Education } \\
\hline Less than high school & $24(3)$ \\
\hline High school & $111(13)$ \\
\hline Some college/technical school & $256(31)$ \\
\hline College & $236(29)$ \\
\hline Graduate training/degree & $196(24)$ \\
\hline Declined to respond & 104 \\
\hline \multicolumn{2}{|l|}{ Employment $^{\mathbf{b}}$} \\
\hline Full-time & $342(42)$ \\
\hline Part-time & $135(16)$ \\
\hline Self-employed & $28(3)$ \\
\hline Home & $31(4)$ \\
\hline Disabled & $56(7)$ \\
\hline Unemployed with benefits & $15(2)$ \\
\hline Unemployed without benefits & $37(5)$ \\
\hline
\end{tabular}

Table. (continued).

Demographic Characteristics of Adult Patients Receiving Osteopathic Manipulative Treatment $(\mathbf{N}=927)^{\mathrm{a}}$

\section{Demographic Characteristic}

\begin{tabular}{lc}
\hline Retired & $186(23)$ \\
\hline Student & $41(5)$ \\
\hline Other & $5(1)$ \\
\hline Declined to respond & 103 \\
\hline Annual Household Income, \$ & $122(21)$ \\
\hline$<25,000$ & $137(23)$ \\
\hline $25,000-49,999$ & $131(22)$ \\
\hline $50,000-74,999$ & $78(13)$ \\
\hline$\quad 75,000-99,999$ & $124(21)$ \\
\hline$\geq 100,000$ & 335 \\
\hline & Declined to respond \\
\hline & \\
\hline & $\begin{array}{l}\text { Percentages are reported based on the number of patients who } \\
\text { responded. Data are given as No. (\%) unless otherwise indicated. } \\
\text { Patients were asked to indicate all employment statuses that } \\
\text { applied. }\end{array}$
\end{tabular}

significant difference was found between the sample and the county population in the percentage of people who were disabled (mean difference, $-0.4 \% ; 95 \% \mathrm{CI}$, $-6.0 \%$ to $5.3 \%$ ). The median annual household income in the sample was significantly higher than that of the county population; 5 clinics (33\%) had a sample median annual household income of $\$ 75,000$ or greater, whereas 1 clinic (7\%) had a county population median income of $\$ 75,000$ or greater.

The top 25 chief complaints reported by patients were primarily pain or discomfort (20 of 25), but headaches, sciatica, and upper gastrointestinal problems (eg, gastroesophageal reflux disease) were also included (Figure 2A). The most common chief complaints were pain or discomfort in the lower back (311 [34\%]) and neck $(277[30 \%])$. A majority $(859$ [93\%]) reported 1 or more chief complaints related to pain, and 311 (34\%) reported 1 or more chief complaints related to something other than pain. Nineteen of the top 25 medical diagnoses were musculoskeletal disorders 


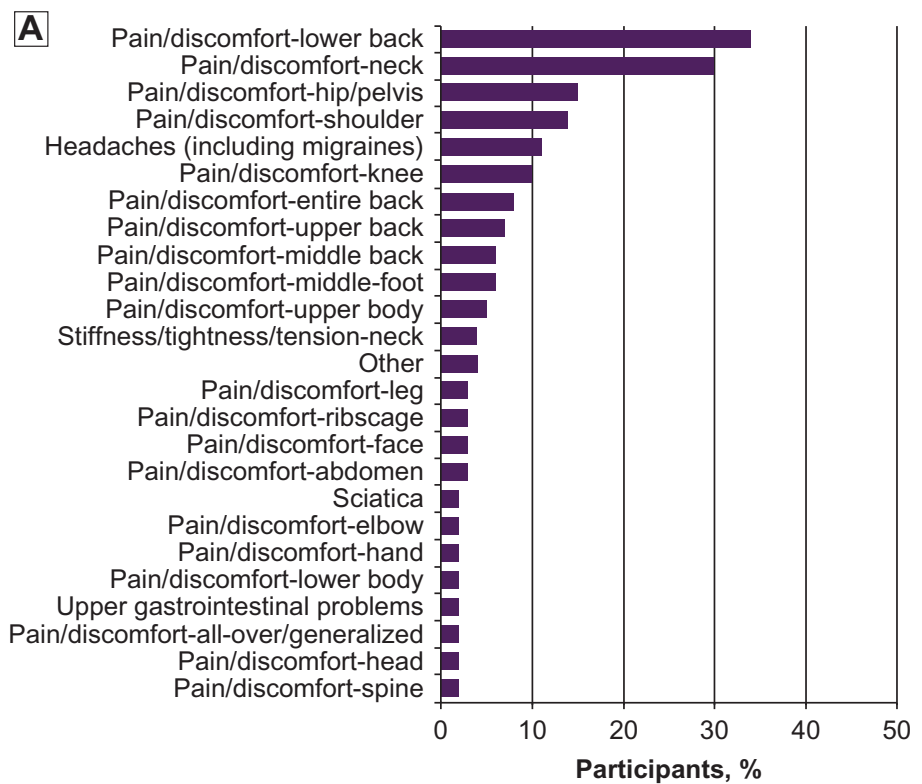

B

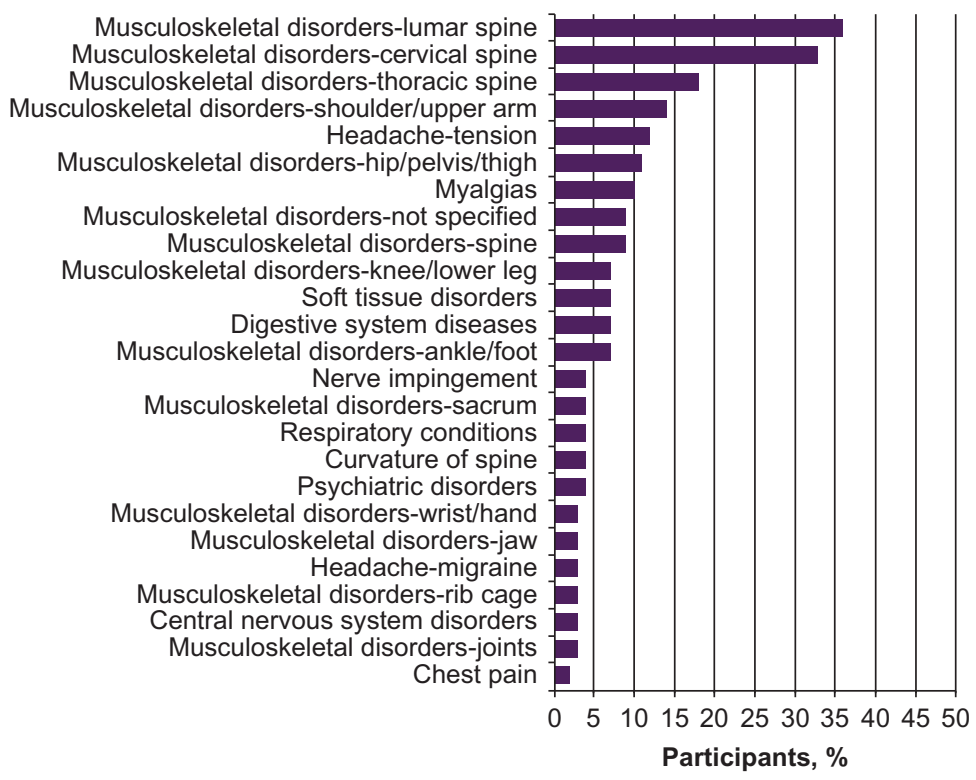

Figure 2.

The top 25 chief complaints and medical diagnoses of adult patients receiving osteopathic manipulative treatment $(\mathrm{N}=927)$. (A) Chief complaints; (B) medical diagnoses.

(Figure 2B). The most common medical diagnoses were musculoskeletal disorders in the lumbar $(334$ [36\%]) and cervical (309 [33\%]) regions.

Of the 927 patients, 807 (87\%) indicated they had used 1 or more of the following treatments and activ- ities for their chief complaints: exercise (612 [66\%]), medication (563 [61\%]), stretching or yoga (532 [57\%]), massage therapy (407 [44\%]), chiropractic adjustment (375 [41\%]), physical therapy (312 [34\%]), supplements (271 [29\%]), acupuncture (104 [11\%]), or 
surgery $(95[10 \%])$. The most helpful (helped quite a bit or very much) treatments or activities patients had used for their chief complaints were OMT (534/647 [83\%]), surgery (53/92 [58\%]), and medication (298/ 549 [54\%]; Figure 3A). The least helpful (did not help at all or made chief complaint worse) treatments or activities were surgery (19/92, [21\%]) and supplements $(54 / 258,[21 \%])$.
Of the 226 patients who were new patients with no previous OMT for their chief complaint(s), 163 (72\%) indicated they had used 1 or more of the following treatments and activities for their chief complaints: exercise (108 [48\%]), stretching or yoga (106 [47\%]), medication (100 [44\%]), chiropractic adjustment (65 [29\%]), massage therapy (61 [27\%]), physical therapy (48 [21\%]), supplements (47 [21\%]), surgery (14
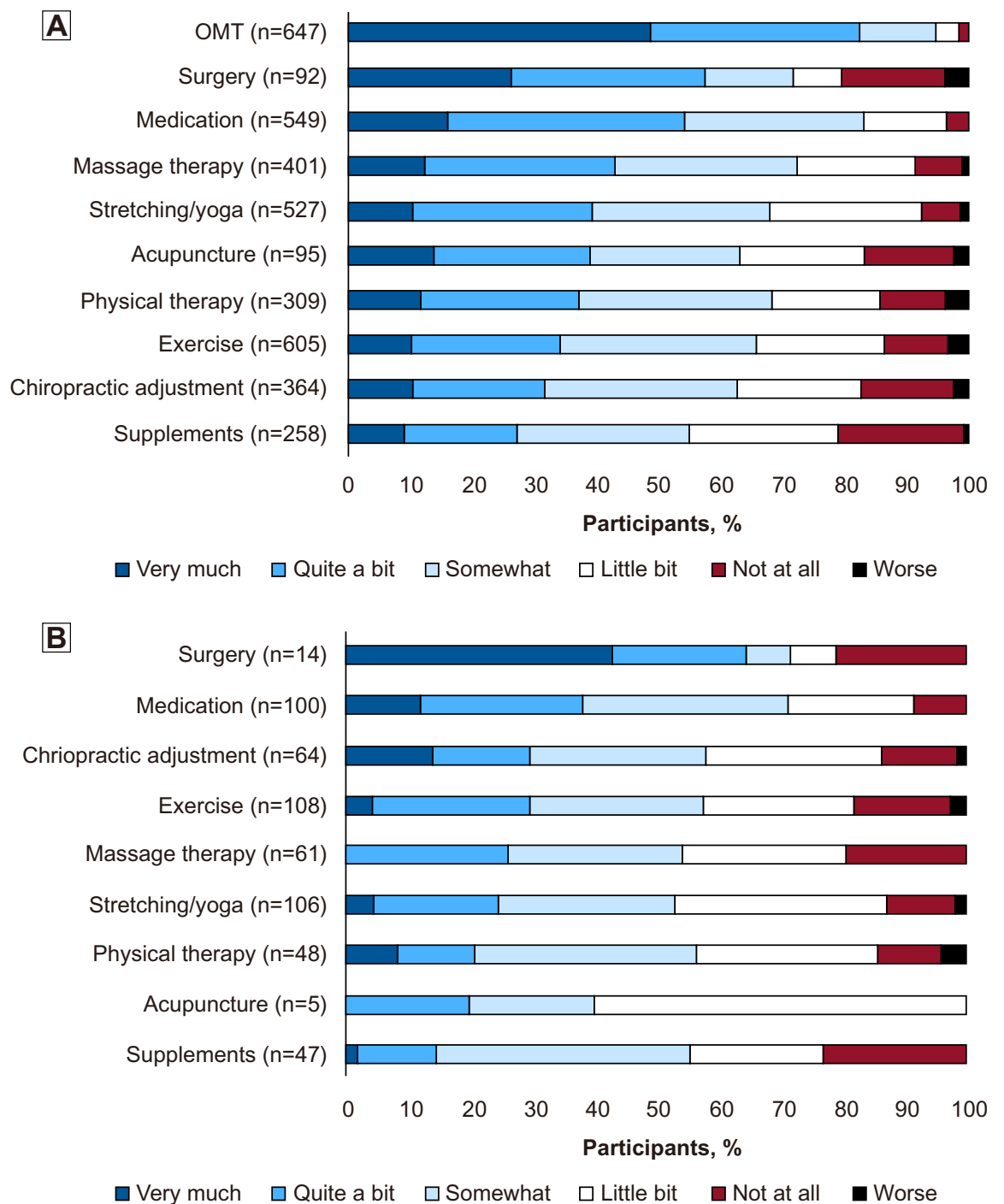

Figure 3.

Helpfulness of previous treatments or activities used to address chief complaint(s) reported by adult patients receiving osteopathic manipulative treatment (OMT), ordered from most helpful to least helpful. (A) All patients (N=927); (B) new patients $(n=226)$. 
[6\%]), or acupuncture (6 [3\%]). The most helpful previous treatments or activities were surgery (9/14 [64\%]), medication $(38 / 100,[38 \%])$, chiropractic adjustment $(19 / 64,[30 \%])$, and exercise $(32 / 108,[30 \%])$, and the least helpful were supplements (11/47, [23\%]), surgery $(3 / 14,[21 \%])$, and massage therapy $(12 / 61,[20 \%]$; Figure 3B).

Before OMT, patients' health-related quality of life $(n=852)$ was significantly worse than that of the general US population (Figure 4). Fatigue (55.9; 95\% CI, 55.4-56.3; 1784 office visits) and pain interference (57.9; 95\% CI, 57.6-58.3; 1784 office visits) $t$ scores were more than $0.5 \mathrm{SD}$ higher than that of the US population. Physical functioning (43.4; 95\% CI, 43.0-43.8;1710 office visits) $t$ scores were more than 0.5 SD lower than that of the US population. Sleep disturbance (52.6; 95\% CI, 52.2-53.0; 1787 office visits) $t$ scores were slightly higher than that of the US population.

\section{Discussion}

Results from the current study indicated that patients receiving OMT were primarily women, white, not Hispanic or Latinx, and middle-to-high socioeconomic status. As such, they were not representative of the county population in terms of age, gender, race, ethnicity, or socioeconomic status. However, these results were mainly consistent with those previously reported for the use of CAM approaches by US adults and the use of manipulative therapies. ${ }^{2,3,7}$

Consistent with results from our preliminary study, ${ }^{9}$ the chief complaints and medical diagnoses of patients receiving OMT were primarily musculoskeletal pain conditions, particularly low back and neck pain, although conditions of the digestive system (eg, gastroesophageal reflux disease) were also common. Similar findings have been reported for practitioner-based therapies in general. ${ }^{3-5} \mathrm{~A}$ more detailed analysis of the use of CAM approaches by US adults with musculoskeletal disorders indicated that people with sciatica, low back pain, and neck pain used practitioner-based therapies as part of their treatment more frequently than those with other musculoskeletal problems (eg, arthritis, other joint conditions). ${ }^{5}$ Of US adults who responded to the 2007 National Health Interview Survey, 6.8\% used chiropractic or osteopathic manipulation for management of illness, and $3.8 \%$ used them for health promotion. ${ }^{1}$ Thus, the continued expansion of DO-Touch.NET's registry of patients receiving OMT is necessary to extend the evidence base for OMT beyond musculoskeletal pain conditions.

The most commonly reported treatments and activities previously used by patients receiving OMT in the current study for their chief complaints were exercise, medication, and stretching or yoga. All treatments and activities that had been previously used were more helpful than not, but substantial numbers of patients reported that prior treatments and activities failed to help with their chief complaints. This finding supports a previous study ${ }^{13}$ that found the second most frequently reported reason for referring patients for CAM was failure of conventional treatments. To our knowledge, information on treatments and activities used prior to OMT are limited to case reports. Therefore, published failure rates for specific treatments and activities may be helpful for patients and clinicians making treatment decisions.

Before treatment, patients who received OMT in the current study had more fatigue, pain interference, and sleep disturbance, and worse physical functioning than the general US population. Palinkas and Kabongo ${ }^{5}$ found primary care patients who used practitioner-based therapies had lower physical functioning and more pain than those who did not use any CAM approaches. In another study, patients presenting for OMT at a single specialty clinic were surveyed about their quality of life using the 36-Item Short Form Health Survey (SF-36). ${ }^{14}$ Patients reported worse physical function, bodily pain, and vitality than the general US population. ${ }^{14}$

PROsetta Stone (www.prosettastone.org) was used to link results from the current study, which used PROMIS measures, to the results reported by Licciardone et $\mathrm{al},{ }^{14}$ which used the SF-36. Results 
A

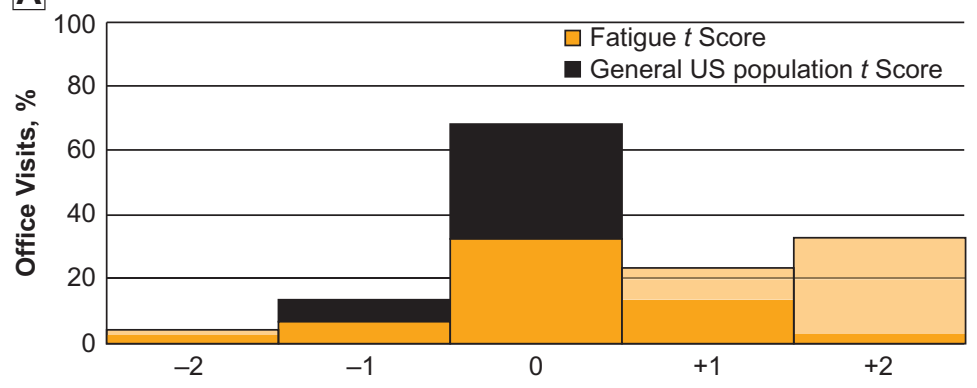

B

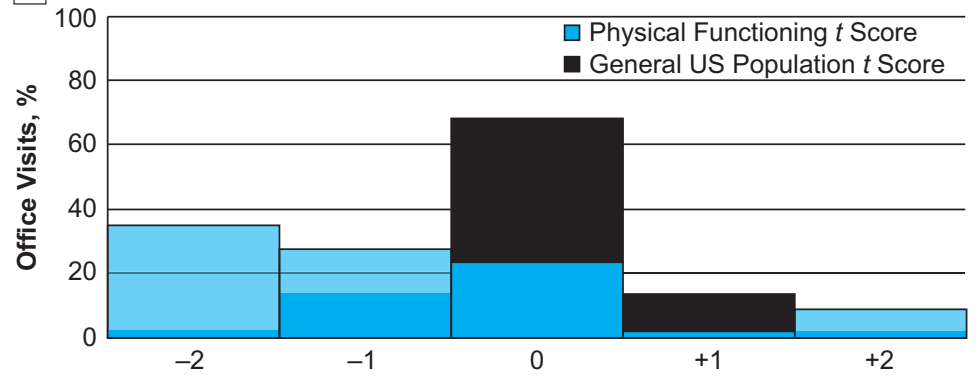

C

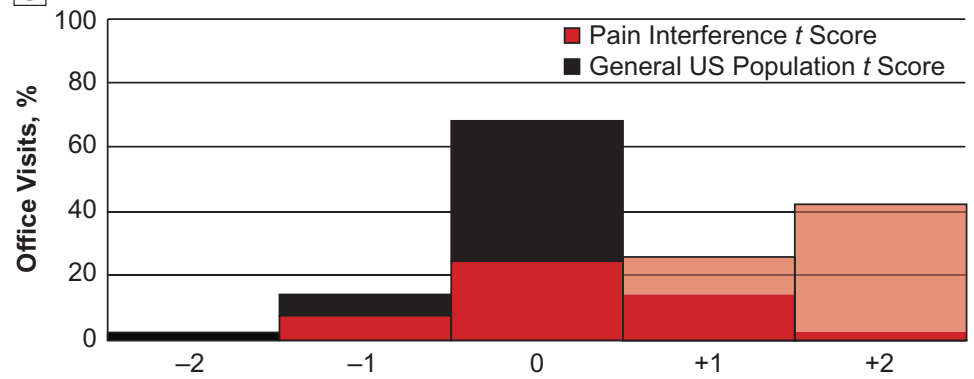

D

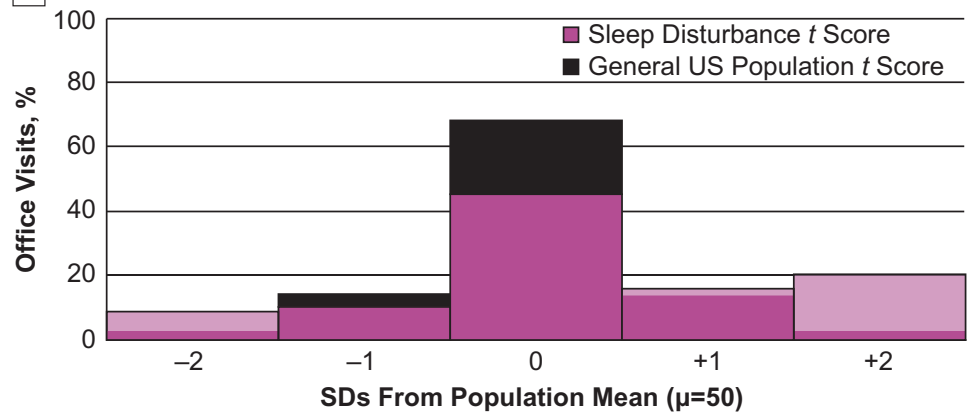

Figure 4.

Health-related quality of life of adult patients receiving osteopathic manipulative treatment compared with the general population of the United States ( $\mathrm{N}=852)$. The general US population has a mean (SD) $t$ score of $50(10) .{ }^{11} t$ scores above 50 indicate worse fatigue, pain interference, sleep disturbance, and better physical functioning than the general US population. (A) Fatigue; (B) pain interference; (C) physical functioning; (D) sleep disturbance. 
from the 2 studies were similar for physical function and pain interference. However, patients from the current study reported worse fatigue than patients from the single-site study. ${ }^{14}$ The patients who sought OMT, most of whom had musculoskeletal pain conditions, had a worse quality of life than the general US population. Future studies that examine the effect of OMT on quality of life, particularly over multiple treatment sessions, are needed to determine whether OMT is effective in restoring quality of life.

The current study, strengthened over previous studies by including data from a large number of patients from multiple and geographically diverse clinics, had several limitations. The comparison between study patients and county populations may have been affected by patients who declined to provide demographics, such as ethnicity (20\%), education (11\%), employment (11\%), and income (36\%), particularly if there was systematic bias in which data were not provided. Furthermore, the patient population for the clinics may have included patients from outside the county where the clinic was located, and the patients may or may not have been representative of the clinic population where they received treatment. Differences between patients who participated and the clinic's general patient population could bias results, limiting the generalizability of the current study. Future studies that prospectively obtain data from all patients at participating clinics would be ideal for a more generalizable view of the characteristics of patients who receive OMT.

Classifying medical diagnoses into homogeneous groupings that consistently distinguished between musculoskeletal and nonmusculoskeletal conditions was complicated by the lack of specificity of the International Classification of Diseases, Ninth Revision codes extracted from patients' medical records. For example, the diagnosis grouping labeled "chest pain" consisted of diagnoses of chest pain not otherwise specified or not elsewhere classified. Since it was unclear whether chest pain in these cases was of musculoskeletal, cardiovascular, or gastrointestinal origin, these diagnoses were classified as a separate grouping. In future studies, the need for specificity in coding diagnoses should be balanced with the time constraints of clinicians.

New and established patients were included in the current study. Although we examined the subgroup of new patients who had not previously used OMT for their chief complaint(s), these patients may have received OMT for other conditions. For patients who had previously used OMT for their chief complaints, a majority reported that OMT helped very much or quite a bit, and only $2 \%$ reported that it did not help at all or made the chief complaint worse. Patients who previously found OMT helpful for their chief complaints may be more likely to repeat the experience or have higher expectations for positive treatment outcomes than those who experienced little to no relief from OMT. Future observational research on OMT should assess patient expectations for treatment outcomes and evaluate their effect on those outcomes. ${ }^{15}$

\section{Conclusion}

Adult patients receiving OMT in the United States are being treated primarily for musculoskeletal pain conditions, are not representative of the population of the county where the clinic is located, have previously used a variety of treatments and activities to address their chief complaints, and generally have worse health-related quality of life than the general US population. Information about the characteristics of patients who use OMT is important for defining osteopathic distinctiveness and identifying potential areas for increased use of OMT. While this is a step toward DO-Touch.NET's goal of expanding the evidence base for OMT, additional research regarding the effectiveness and safety of OMT as it is used in the clinical setting is necessary to determine whether there are identifiable patient characteristics that are related to patients' response to OMT.

\section{Author Contributions}

Both authors provided substantial contributions to conception and design, acquisition of data, or analysis and interpretation 
of data; both authors drafted the article or revised it critically for important intellectual content; both authors gave final approval of the version of the article to be published; and both authors agree to be accountable for all aspects of the work in ensuring that questions related to the accuracy or integrity of any part of the work are appropriately investigated and resolved.

\section{Acknowledgments}

We appreciate the project management support provided by Lisa Norman, BS, PMP, and the editorial support provided by Deborah Goggin, MA, ELS, from Research Support at A.T. Still University. Additionally, we appreciate the insightful review by Cheryl B. Schwartz, DO, PhD, private practice. We also acknowledge the support of DO-Touch.NET members and their staff from the following clinics who actively participated in the study by providing access to and data on their patients: A.T. Still University Osteopathic Manipulative Medicine Clinic, Kirksville, MO; Bee Caves Family Practice, Lakeway, TX; BMS Integrated Health, Farmington Hills, MI; William J. Brooks, DO, Kansas City, MO; Capital Region Medical Center, Jefferson City, MO; Crossroads Premiere Health Care, Corvallis, OR; Feely Center for Optimal Health, Chicago, IL; Jefferson Park Medical Group, Chicago, IL; Kirksville Family Medicine, Kirksville, MO; Kohn Group, McHenry, IL; Midwestern University Multispecialty Clinic, Downers Grove, IL; Midwestern University Multispecialty Clinic, Glendale, AZ; New Medical Health Care, Wichita, KS; Osteopathic Center of the Four Corners, Durango, CO; Rocky Mountain Integrative Medicine, Ridgway, CO; Elizabeth M. Sasaki, DO, Visalia, CA; and Walker Family Medicine, Winchester, TN.

\section{References}

1. Davis MA, West AN, Weeks WB, Sirovich BE. Health behaviors and utilization among users of complementary and alternative medicine for treatment versus health promotion. Health Serv Res. 2011;46 (5):1402-1416. doi:10.1111/j.1475-6773.2011.01270.x

2. Rafferty AP, McGee HB, Miller CE, Reyes M. Prevalence of complementary and alternative medicine use: state-specific estimates from the 2001 Behavioral Risk Factor Surveillance System. Am J Public Health. 2002;92(10):1598-1600.

3. Hurwitz EL. Epidemiology: spinal manipulation utilization. J Electromyogr Kinesiol. 2012;22(5):648-654
4. Palinkas LA, Kabongo ML. The use of complementary and alternative medicine by primary care patients. a SURF*NET study. J Fam Pract. 2000;49(12):1121-1130

5. Clarke TC, Nahin RL, Barnes PM, Stussman BJ. Use of complementary health approaches for musculoskeletal pain disorders among adults: United States, 2012. Natl Health Stat Report. 2016;(98):1-12.

6. McEachrane-Gross FP, Liebschutz JM, Berlowitz D. Use of selected complementary and alternative medicine (CAM) treatments in veterans with cancer or chronic pain: a cross-sectional survey. BMC Complement Altern Med. 2006;6(1):34. doi:10.1186/1472-6882-6-34

7. Clarke TC, Black LI, Stussman BJ, Barnes PM, Nahin RL. Trends in the use of complementary health approaches among adults: United States, 2002-2012. Natl Health Stat Report. 2015;(79):1-16.

8. Licciardone JC, Herron KM. Characteristics, satisfaction, and perceptions of patients receiving ambulatory healthcare from osteopathic physicians: a comparative national survey. J Am Osteopath Assoc. 2001;101(7):374-385.

9. Degenhardt BF, Johnson JC, Gross SR, Hagan C, Lund G, Curry WJ. Preliminary findings on the use of osteopathic manipulative treatment: outcomes during the formation of the practice-based research network, DO-Touch.NET. J Am Osteopath Assoc. 2014;114(3):154-170.

10. Harris PA, Taylor R, Thielke R, Payne J, Gonzalez N, Conde JG. Research electronic data capture (REDCap): a metadata-driven methodology and workflow process for providing translational research informatics support. J Biomed Inform. 2009;42(2):377-381.

11. Cella D, Riley W, Stone A, et al. The Patient-Reported Outcomes Measurement Information System (PROMIS) developed and tested its first wave of adult self-reported health outcome item banks: 2005-2008. J Clin Epidemiol. 2010;63(11):1179-1194.

12. United States Census Bureau. American FactFinder: 2010 Census. Suitland, MD: United States Census Bureau; 2010. https://factfinder census.gov/faces/nav/jsf/pages/community_facts.xhtml. Accessed June 6, 2017

13. van Haselen RA, Reiber U, Nickel I, Jakob A, Fisher PA. Providing complementary and alternative medicine in primary care: the primary care workers' perspective. Complement Ther Med. 2004;12(1):6-16.

14. Licciardone JC, Gamber RG, Russo DP. Quality of life in referred patients presenting to a specialty clinic for osteopathic manipulative treatment. J Am Osteopath Assoc. 2002;102(3):151-155.

15. Licciardone JC, Russo DP. Blinding protocols, treatment credibility, and expectancy: methodologic issues in clinical trials of osteopathic manipulative treatment. J Am Osteopath Assoc. 2006;106(8):457-463.

() 2019 American Osteopathic Association 